\title{
Monitoring of Physical Processes in Upper Atmosphere, lonosphere and Magnetosphere in lonosphere Space Missions
}

\author{
Anatoly Petrukovich ${ }^{1}$, Mikhail Mogilevskii ${ }^{1}$, Igor Kozlov ${ }^{1}$, Sergey Pulinetz ${ }^{1}$, Yurii \\ Dobrolenskii ${ }^{1}$, Konstantin Anufreichik ${ }^{1}$, Dmitrii Chugunin ${ }^{1}$, Vladimir Nazarov ${ }^{1}$, Aleksandr \\ Abbakumov ${ }^{1}$, Vladimir Kuznetsov ${ }^{2}$, Stanislav Andrievskii ${ }^{2}$, Yurii Mikhailov $^{2}$, \\ Mikhail Panasyuk ${ }^{3,4}$, Sergey Svertilov ${ }^{3,4^{*}}$, Ivan Yashin ${ }^{3}$, Vitaly Bogomolov ${ }^{3,4}$, Anatoly \\ Iyudin $^{3}$, Vladimir Kalegaev ${ }^{3,4}$, Vladislav Osedlo ${ }^{3}$, Vasilii Petrov ${ }^{3}$, Andrey Shemukhin ${ }^{3}$, \\ Aleksey Repin ${ }^{5}$, Nadezhda Kotanaeva ${ }^{5}$, Pavel Moiseev ${ }^{6}$, Sergey Poljakov ${ }^{7}$, Yurii Shlugaev ${ }^{7}$, \\ Vasilii Asmus ${ }^{8}$, Sergey Uspenskii ${ }^{8}$, Leonid Makridenko ${ }^{9}$, Aleksandr Gorbunov ${ }^{9}$ and \\ Vladimir Kozhevnikov ${ }^{9}$ \\ ${ }^{1}$ Institute of Space Research, Russian Academy of Science, 117997, Profsoyuznaya st. 84/32., \\ Moscow, Russia \\ ${ }^{2}$ N.V. Pushkov Institute of Terrestrial Magnetism, Ionosphere and Radio-wave Propagation, Russian \\ Academy of Science, 108840, Kaluzhskii road 4, Troitsk, Moscow, Russia \\ ${ }^{3}$ M.V. Lomonosov Moscow State University, D.V. Skobeltsyn Institute of Nuclear \\ Physics, 119991, Leninskie Gory 1(2), GSP-1, Moscow, Russia \\ ${ }^{4}$ M.V. Lomonosov Moscow State University, Physics Department, 119991, Leninskie Gory, \\ Moscow, Russia \\ ${ }^{5}$ E.K. Fedorov Institute of Applied Geophysics, 129128, Rostokinskaja st. 9, Moscow, Russia \\ ${ }^{6}$ Astron Electronics, 302019, Veselaya st. 2, Orel, Russia \\ ${ }^{7}$ Nizhnii Novgorod State University, Radio-physical Institute, 603950, Bolshaya Pecherskaja st. 25, \\ Nizhnii Novgorod, Russia \\ 8 Scientific and Research Center of Space Hydro-meteorology Planeta, 123242, Bolshoy \\ Predtechenskii alley 7, Moscow, Russia \\ ${ }^{9}$ VNIIEM Corporation, 107078, Horomny tupik 4, 1, Moscow, Russia
}

\begin{abstract}
The Ionosphere missions are the part of Ionosond-2025 space project, which main scientific objectives are monitoring of physical processes in the Earth upper atmosphere, ionosphere and magnetosphere, as well as of solar activity. Within the framework of the Ionozond-2025 project, it is planned to launch four spacecraft Ionosphere and one satellite Zond. The Zond satellite is planned to be launched in 2025. The main task of Zond mission is patrol of solar activity. The launch of the first pair of spacecraft is planned in early 2022, the second pair - late 2022 or early 2023. In case of successful implementation of the program of experiments on the Ionosphere satellites, control of the physical parameters of electromagnetic fields and corpuscular radiation in the near-Earth space will be provided, new information will be obtained on the geophysical processes occurring in the magnetosphere, ionosphere and upper atmosphere in their connection with solar activity.
\end{abstract}

\footnotetext{
*Corresponding author: sis@coronas.ru
} 


\section{Introduction}

As it is well-known, the ionosphere is the upper layer of the atmosphere, located above about $85 \mathrm{~km}$, the matter of which is strongly ionized mainly by ultraviolet and X-ray radiation from the Sun. The ionosphere plays an important role in the propagation of radio waves, therefore its monitoring is essential to ensure the quality of radio communications. In addition, the ionosphere can be considered as a natural laboratory, in which the processes of interaction of plasma and waves can be studied directly by the in situ measurements. This causes a great interest in ionospheric research, which has been intensively carried out over the past decades both by ground, air (rockets, high-altitude balloons) and orbital instruments.

Despite that to date, extensive experimental material has been accumulated about the ionosphere and theoretical models have been developed that make it possible to describe its states in significant details, there are still urgent research problems associated with the need to study those regions of the ionosphere in which our knowledge (and models) do not allow us to confidently predict the parameters of the ionospheric plasma. These areas include primarily the auroral and equatorial regions.

The peculiarities of the auroral region are that the classical mechanisms of plasma formation, based on the effects associated with exposure to solar radiation, do not work in it. Therefore, it is assumed that the key role in its formation (especially during the polar night) magnetosphere tail particle precipitation are played. As a result, the $\mathrm{F}$ layer is strongly weakened (or disappears) and the E layer plays a decisive role. Therefore, the problem of the parameters of the auroral ionosphere becomes "volumetric", the solution of which depends on the state of the magnetosphere and the features of precipitation. As a result, the "quality" of the ionosphere also changes, i.e. it becomes highly heterogeneous and non-stationary.

The equatorial region also differs from the mid-latitude region due to the so-called floating "bubbles" that violate the generally accepted structure.

A separate range of tasks in the study of non-stationary processes in the ionosphere is based on the use of artificial disturbances of the ionosphere by impact of high-frequency (HF) electromagnetic radiation generated by heating stands (Sura, Tromsö, HAARP and others), low-frequency (LF) radiation from powerful ground-based transmitters, as well as disturbances arising from the influence of electric circuit harmonics. These studies have a "double" meaning. On the one hand, it is controlled effect on the ionospheric plasma, which should make it possible to simulate the phenomena occurring in nature, but with known exposure parameters. On the other hand, there is number of physical problems for which "unlimited" conditions for carrying out measurements are important (the dimensions of the measuring instruments are much smaller than all the characteristic dimensions in plasma) and such measurements can be implemented in a kind of "space laboratory", which in fact, the ionosphere is.

To solve these problems, an integrated approach is required based on the use of instruments that provides detection of different types of radiation, as well as spatially separated measurements based on a satellite system. This approach lies at the heart of the Ionosphere space mission goals.

\section{Concept of space missions lonosphere}

The main tasks of the Ionosphere space missions include:

- monitoring of the ionosphere;

- monitoring of the magnetosphere;

- measurement of wave activity (electromagnetic, acoustic waves); 
- diagnostics of corpuscular ionizing radiation;

- diagnostics of the Atmosphere ozone (determination of the planetary ozone distribution).

Ionosphere satellites should be launched into near-Earth orbits with a set of instruments to monitor the environmental helio-geophysical parameters in the upper atmosphere, ionosphere and near-earth space. Spacecraft "Ionosphere" should operate in a near-circular sun-synchronous orbits in two orbital planes ( 2 spacecraft in each plane). The spacecraft in each plane should be separated by an angle of $180 \pm 30^{\circ}$. Orbital parameters of the first pair of spacecraft: altitude $\sim 820 \mathrm{~km}$; orbital period $\sim 101 \mathrm{~min}$; inclination $\sim 98^{\circ}$; eccentricity $\sim 0.001$; the position of the orbital plane relative to the mean Sun right ascension $\sim 135^{\circ}$; the local time of the orbital ascending node is $\sim 21 \mathrm{~h}$. The orbital parameters of the second pair of spacecraft differ in the position of the orbital plane relative to the mean Sun right ascension - $\sim 6^{\circ}$ and the local time of the orbital ascending node $-\sim 15 \mathrm{~h}$. The launch of the first pair of spacecraft is planned in early 2022, the second pair - late 2022 or early 2023.

Scientific payload includes instruments for radio tomography of the upper atmosphere and ionosphere:

- ionozond for the range 0.1 - $20 \mathrm{MHz}$ with a $137 \mathrm{MHz}$ transmitter (LAERT instrument);

- Langmuir probe and ionospheric plasma energy spectrometer (ESIP instrument);

- GPS - GLONASS meter of total electron content (TES instruments);

- VLF wave complex of instruments;

- onboard radio transmitters at frequencies of $150,400 \mathrm{MHz}$ (equipment Mayak $150 / 400)$;

- hard X-ray and gamma ray spectrometer (SG/1 instrument);

- spectrometer of plasma and energetic charged particles (SPER/1 instrument);

- detector of galactic cosmic rays (GALS/1 instrument);

- ozonometer;

- onboard complex for payload control and scientific data collection (BKUSNI);

- system for transmitting output data from the antenna-feeder device of the target information radio link (AFU RLCI).

Thus, scientific payload includes five groups of instruments: instruments for ionosphere plasma parameters monitoring, instruments for electromagnetic wave monitoring, instruments for magnetosphere particle and space weather monitoring, ozonometer and instruments for data collecting and transmitting. The payload composition of all four Ionosphere spacecraft is the same, except that the GALS/1 instruments and the ozonometer will only be installed on the second pair of spacecraft.

The key instrument of the ionospheric group of equipment is the ionospheric locator, i.e. the LAERT ionozond, which allows measuring the ionospheric plasma density profiles. The ionozond measurements can be used to reconstruct the height distribution of the electron concentration up to the height of the F2 layer maximum. These measurements are remote, and their interpretation can be carried out both on board the spacecraft and after receiving telemetry (TM) information in ground-based laboratories. The second approach is most used for complex distributions of electron concentration and in non-stationary geomagnetic conditions.

Another approach to determining the electron concentration at significant distances from the measurement site is based on the use of signals from navigation satellites such as GPS/GLONASS. This method makes it possible to determine the concentration of electrons in the column along the signal propagation path from the source (GPS/GLONASS satellite) to the receiver of the Ionosphere spacecraft. 
Accurate measurements of the electron concentration and temperature of particles at the spacecraft location point carried out using a Langmuir probe and an ionospheric energy spectrometer.

To determine small-scale ionospheric irregularities, their dynamics and build a threedimensional picture of the plasma distribution, it will be carried out using onboard transmitters "Mayak". The signals from this device will be received on the Earth's surface and processed by the tomographic method.

To determine the distribution of ozone, a simple ozonometer will be used to determine the density of the gas below the satellite.

The SPER/1 instrument is designed to determine the epithermal plasma and energetic charged particle fluxes parameters, which is especially important in the auroral region, where the properties of the ionosphere are largely determined by streams of precipitating particles.

The GALS/1 instrument is designed to measure the fluxes of galactic cosmic rays.

The SG/1 gamma spectrometer is designed to detect the bremsstrahlung of energetic electrons generated in high-altitude electromagnetic discharges, as well as to monitor solar activity in the hard X-and gamma ray ranges and to measure atmospheric albedo fluxes.

The organization of the payload operation on spacecraft board is provided with the help of the acquisition and control device, i.e. BKUSNI. Target data will be transmitted via a special telemetry system.

\section{Monitoring of ionosphere by plasma density measurements}

\subsection{Measurements of electron concentration global distribution by sounding method with the use of LAERT instrument}

The LAERT instruments is designed for global operative monitoring of the Earth's ionosphere by the method of pulsed multi-frequency radio sounding in the frequency range from 0.1 to $20 \mathrm{MHz}$. The LAERT ionozond provides an operative determination of the critical frequency of the ionosphere along the satellite orbit, the calculation of vertical profiles of the electron concentration, the determination of the parameters of the ionospheric plasma at the altitude of the spacecraft orbit using the plasma resonance frequencies. According to these parameters, it is possible to construct a 3-dimensional global distribution of electron concentration in the Earth's ionosphere, to determine the boundaries of the main formations in the ionosphere (equatorial anomaly, main ionospheric trough, auroral oval and polar cap), which affect the propagation of radio waves in various frequency ranges. The ionozond also has the ability to synchronize with ground-based ionospheric stations for conducting sessions of trans-ionospheric and reverse ionospheric sounding.

The LAERT ionozond includes:

- transmitter;

- onboard receiving device;

- signal processing unit;

- transmitting antenna;

- receiving antenna;

- preamplifiers.

The transmitter is designed to emit radio pulses in the frequency range $0.1-20 \mathrm{MHz}$. It includes: broadband amplifier, antenna switch, broadband matching transformer, cable set. 
Main parameters of the transmitter:

- the range of radiated frequencies is $0.1-20 \mathrm{MHz}$;

- input impedance $50 \mathrm{Ohm}$; - output impedance 2x300 Ohm;

- input signal power $0 \mathrm{dBm}$ - power at the antenna input $280 \mathrm{~W}$.

The onboard receiver provides two-channel reception of signals emitted by the transmitter of the satellite ionozond and reflected from the ionosphere; shaping the excitation signal of the transmitting device; formation of a highly stable frequency signal for a central processing unit (CPU).

The on-board receiving device has the following technical parameters:

- the range of received frequencies is from 0.1 to $20 \mathrm{MHz}$;

- bandwidth $(-3 \mathrm{~dB})-15 \mathrm{kHz}$;

- sensitivity (with a signal-to-noise ratio of $10 \mathrm{~dB}$ ) not less than $5 \mu \mathrm{V}$;

- the frequency range of the excitation signal of the power amplifier is from 0.1 to 20 MHz.

During the instrument operation, the number of sounding frequencies and the frequency step are determined by the programmed operating mode. The sounding of the ionosphere is carried out by radio pulses with duration of $100 \mu$ s with a repetition rate of $60 \mathrm{~Hz}$. The standard time for obtaining an ionogram at a frequency of sending probing pulses of $60 \mathrm{~Hz}$ and 400 probing frequencies is 6.7 seconds. The session time is reduced to 5 seconds with the adaptive selection of the frequency grid of sounding. For special measurement modes, a longer ionogram recording period can be provided.

The periodicity of sounding sessions is 10, 30, 60 seconds (set by the commands of the command and control unit (SCU)), while the sounding mode with a frequency of 10 seconds is considered the main one.

There are three main modes of ionozond operation:

- full sweep 0.1 - $20 \mathrm{MHz}$;

- adaptive mode, the ionozond itself determines the critical frequency according to a special algorithm, and in accordance with this, the maximum sweep frequency is set;

- special mode, it is determined by the programmed, it can be work at fixed frequencies, work for reception without a transmitter, work in synchronization mode with a ground ionozond, work in trans-ionospheric sounding mode, etc.

\subsection{Measurement of the ionosphere electron concentration altitude distribution by the method of radio occultation estimation of the amplitude and phase delays of the signals from the global navigation systems GPS/GLONASS with the use of the PES instrument}

With the use of PES instrument, the following main tasks should be solved:

- global monitoring of the structure and dynamics of the ionosphere;

- detection of anomalous phenomena and characteristic signs of changes in the ionosphere, caused by the processes of preparation of earthquakes and man-made influences;

- development of technical means and methods for monitoring natural and man-made disasters from the spacecraft. 


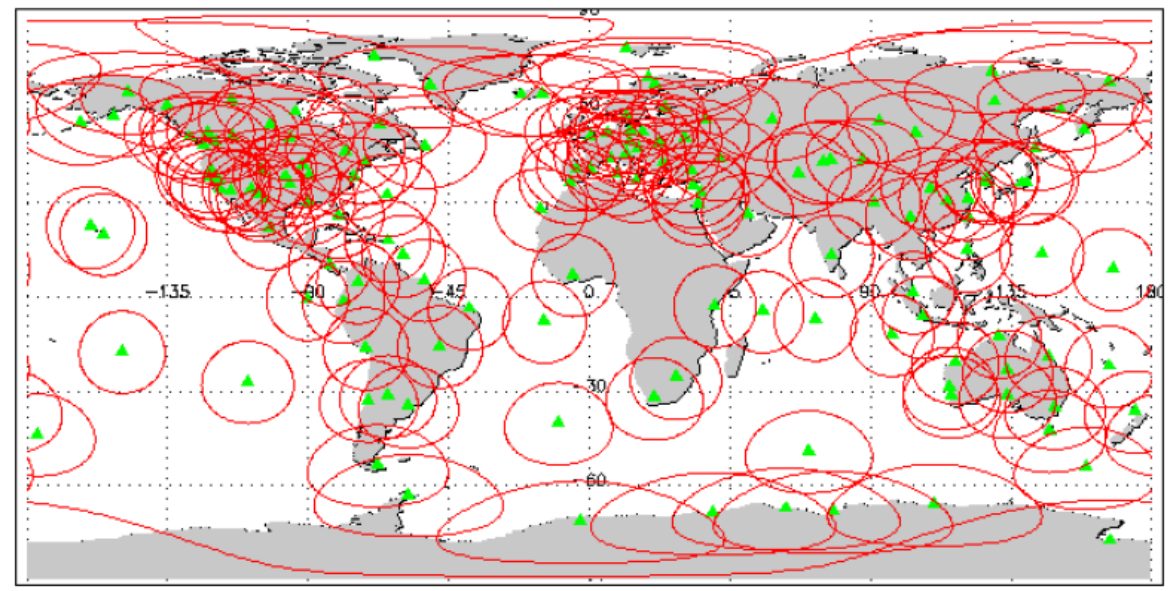

Fig. 1. Global network of ground GPS receivers. The vision areas at $400 \mathrm{~km}$ altitudes at the elevation angle $10^{\circ}$ are shown.

As it is well-known, higher requirements for positioning accuracy have led to the creation of new generation navigation satellite systems GPS-NAVSTAR (NAVSTAR Navigational Satellite Time and Ranging) and GLONASS (Russian Global Navigation Satellite System). The satellites of these systems have a circular semi-synchronous orbit with the Earth's rotation with an altitude of about 20 thousand $\mathrm{km}$ (orbital period 12 hours) and are placed in the indicated orbits in three planes spaced 120 degrees apart. There are 45 satellites in the radio visibility zone of each point on Earth at the same time. The duration of observation of one satellite reaches six hours and, therefore, provides much more information on the spatial and temporal changes in the parameters of the ionosphere.

Currently, the global network of ground-based GPS receivers (see Fig. 1) supplying observational data to regional centers, accessible through public Internet sites, numbers about 1000 points

Continuous high-precision phase-difference measurements at GPS frequencies (1.2 / 1.6 $\mathrm{GHz}$ ) behind all satellites in the field of view at each receiving point of the global network allow obtaining information on daily variations in the total electron abundance in the ionosphere above the point of observation. The processing of measurement data from a network of $\sim 100$ GPS receivers (GDGPS system of global differential corrections) makes it possible to obtain practically on-line distribution of TEC (Total Electron Content) in the near-Earth ionospheric plasma.

Ionospheric correction is transmitted to users of single frequency GPS receivers in order to achieve similar accuracy to users of dual frequency receivers. The GDGPS system reconstructs in real time a global map of ionospheric electronic content (currently the map is updated every 5 minutes). In addition, according to the data of the global network of GPS receiving points, it is possible to study the morphology and dynamics of medium- and large-scale inhomogeneous structures, which are manifested in variations in the total electron content [1].

Recently, special GPS receivers have been produced that allow measuring the amplitude of navigation signals $1.2 / 1.6 \mathrm{GHz}$ with a sampling frequency (up to $50 \mathrm{~Hz}$ ) required for recording radio measurements. With the use of such receivers, strong scintillations of GPS signals were recorded not only in the equatorial zone, but also at mid-latitudes during a moderate magnetic storm [2].

Receiving radio signals from GPS/GLONASS global navigation systems using equipment installed on LEO satellites makes it possible to implement measurement conditions in which a change in the electron concentration integral along the path of radio 
wave propagation is due to a change in the path length and the addition (or exclusion) of a new high-altitude ionospheric layer. These conditions arise during "radio ascents" and "radio calls" of satellites of navigation systems with respect to the low-orbit spacecraft and the Earth, when it is possible to measure the time delay and phase of GPS/GLONASS signals when the lowest point crosses the ionospheric altitude signal propagation path in the interval from $\approx 50 \mathrm{~km}$ to the orbital altitude of a low-orbit spacecraft.

The geometry of radio occultation measurements is shown schematically in Fig. 2.

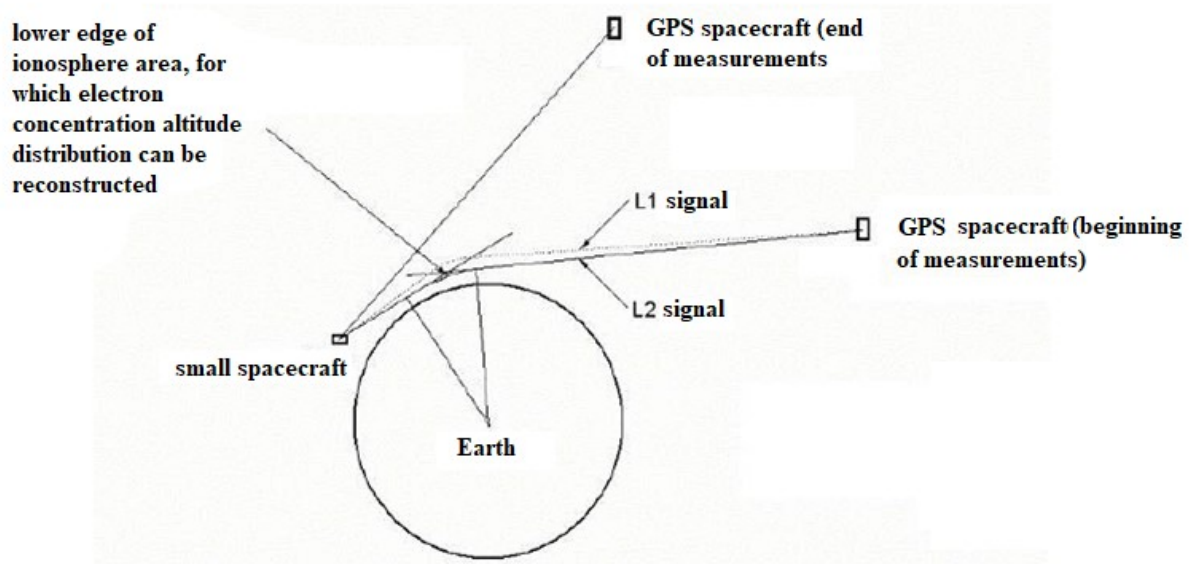

Fig. 2. The scheme of radio occultation measurements, on which there are presented the mutual position of low orbital small spacecraft and satellite of GPS navigation system.

It follows from Fig. 2 that for the conditions of the daytime mid-latitude undisturbed ionosphere for an altitude interval of $100-1000 \mathrm{~km}$, the electron concentration values calculated from the data of radio occultation measurements practically coincide with the model values.

When signals are received on board the spacecraft from satellites of the GLONASS and GPS navigation systems, it is simultaneously possible (with continuous operation of the TES equipment) to measure more than a thousand altitude profiles of the electron concentration of the Earth's ionosphere during the day, i.e., to carry out a global survey of the ionosphere. The measurement program allows you to control the state of the ionosphere over specified regions, including seismically active ones.

Signals from GPS/GLONASS satellites are received by the antenna, filtered and amplified by an antenna amplifier, which is integrated into the antenna housing. Signals of two navigation frequencies come from the antenna to the input of the navigation receiver. In the navigation receiver, the frequency conversion of the input signals and distribution over the tracking channels are performed. Tracking is controlled by the processor. After capturing the satellite channel, the received signal is demodulated in order to extract from it a modulating message about the code phase, carrier phase, satellite clock and navigation message.

The navigation message contains information about the current state of the satellite transmitting the signal, its ephemeris, the state of the GPS/GLONASS systems, the time and almanac of the movement of the satellites. The carrier phase and code phase measurements are sent to the input/output $(\mathrm{I} / \mathrm{O})$ controller. The $\mathrm{I} / \mathrm{O}$ controller provides temporary storage of measurement results and automatic control of the navigation receiver operating mode, in accordance with the measurement program. The controller sets the operating mode of the navigation receiver after the supply voltage is applied, receives and 
executes control commands and transmits the measurement results via the serial data interface.

Switching the operating modes of the TES equipment is carried out in accordance with a given program, taking into account the spatial position of the spacecraft relative to the satellites of the GPS/GLONASS navigation systems, its geographic position and current time. The measurement results are tested for compliance with the established criteria in order to transfer only useful data to the satellite board systems.

The antenna of the PES equipment is active and provides reception of signals in two frequency ranges 1217 - $1265 \mathrm{MHz}$ (L1) and 1565 - $1615 \mathrm{MHz}$ (L2).

The navigation receiver contains a high-frequency linear and digital part. The highfrequency linear part of the receiver operates in the input frequency ranges L1, L2 and is designed to transfer the spectra of the received GPS signals to the video frequency range.

\subsection{Radio-interferometric methods of diagnostics of the ionosphere with the use of Mayak 150/400 instruments}

Radio interference methods were developed by the Soviet radio-physical school, created by academicians L.I. Mandelstam and N.D. Papaleksi [3, 4]. The use of these methods for measuring the regular and inhomogeneous structure of the electron concentration in the near Earth surface and space plasma using geophysical rockets, satellites and interplanetary spacecraft has shown their effectiveness and formed an independent radio-physical research direction, i.e. radio beacon methods for diagnosing the environment. The name of these methods is associated with the name of the first coherent transmitters of VHF radio waves "Mayak", which were installed on research rockets. Since 1957 coherent transmitters "Mayak" in various modifications are widely used in space experiments on LEO spacecraft, on geostationary satellites and on interplanetary spacecraft.

The consistent development of satellite radio beacon methods ultimately led to a technological breakthrough, i.e. to the creation of space systems for high-precision temporal and spatial positioning - first the first-generation low-orbit radio navigation systems, and then high-orbit GPS and GLONASS systems.

The physical foundations of the radio beacon method are quite simple and clear $[5,6]$. The beacon radio transmission method is based on the fact that when radio waves propagate in a collisional anisotropic partially ionized inhomogeneous plasma, which is the Earth's ionosphere, radio waves interact with the propagation medium due to known effects, such as refraction, dispersion, absorption, scattering on fluctuations of electron density, etc.

As a result of this interaction, the radio waves received on Earth contain information about the parameters of the sub-satellite region, incl. information about the regular, wave and stochastic structure.

The organization and geometry of radio beacon measurements determines the set of environmental parameters determined from ground-based registration data. The most used options for using radio beacon methods (depending on which facility the radio beacon is installed on):

- rocket experiments - provide high-precision measurements of the height profile of the electron concentration from the base of the ionosphere to the height of the rocket ascent; the concentration profile is determined both in the ascent and in the descent of the rocket [7];

- low-orbit satellites - provide measurement of the space-time structure of the subsatellite ionosphere (total electron content, TEC), horizontal TEC gradients, as well as spectra of ionospheric irregularities (with synchronous measurements using receiving antennas with a small separation base of $\sim 500-1000 \mathrm{~m}$ ) [8];

- geostationary satellites - high-precision measurements of TEC and diurnal variations of the sub-satellite thickness of the ionosphere and magnetosphere along the fixed direction 
"SC - receiver"; measurements of this kind make it possible to diagnose the impact of powerful ground-based HF stands on the near-earth plasma [9];

- radio occultation measurements - the geometry of "radio sunrise" and "radio entry" is used for radio beacon studies of the ionospheres of planets of the solar system (Venus, Mars) using interplanetary spacecraft [10], as well as for measuring the altitude profiles of the Earth's atmosphere and ionosphere - when the reception on low-orbit satellites of radio beacon signals from the spacecraft of the GPS/GLONASS navigation constellation [11].

The most widespread are radio beacon measurements for the diagnosis of the ionosphere in natural conditions and under anthropogenic impacts using low-orbit satellite radio navigation systems (SRNS), which are equipped with transmitters emitting two coherent signals with frequencies of 150 and $400 \mathrm{MHz}$. On the network of ground receiving stations, the amplitudes and Doppler shift of the received radio navigation signals are measured, time stamps and navigation messages are allocated, and the regular (total electron content in the sub-satellite region of the ionosphere) and statistical scintillation characteristics characterizing the space-time parameters of inhomogeneities in the subsatellite region of the ionosphere are calculated.

Radio-beacon measurements on LEO spacecraft are usually organized according to the following scheme:

- a radio beacon (transmitter) is installed on board the spacecraft;

- the radiation pattern of radio beacon signals in the alignment of angles $\pm 60^{\circ}$ from the direction to the nadir ensures their reception on the Earth within the line of sight of the spacecraft;

- the radio beacon is switched on either in continuous radiation mode, or operates according to a cyclogram, which provides the ability to receive radio beacon signals at "dedicated" ground receiving points, equipped with the necessary complex of antenna and specialized receiving equipment;

- with appropriate organization of radio beacon measurements on a "chain" of (4-6) ground receiving points, a radio-tomographic method of data processing can be implemented, which gives the most complete reconstruction of the spatial structure of the ionosphere over a given region.

Radio-tomography of the ionosphere based on LEO signals using 150/400 MHz radio beacons makes it possible to reconstruct the two-dimensional distribution of electron concentration in the sub-satellite ionosphere [12 - 14]. The applied phase-difference measurement method has no drawback associated with the problem of determining the unknown constant $2 \pi \mathrm{n}$ of the total phase of the signal. In contrast to phase tomography, the phase-difference method is more sensitive to relatively small inhomogeneities of the electron density, which make a small contribution to the total phase, but more significant to its derivative. The reconstructions of the electron density field, performed using the phase tomography method, do not show details less than a few hundred $\mathrm{km}$ in size, while phasedifference tomography allows the reconstruction of ionospheric structures $\sim 100 \mathrm{~km}$ in size and the value of electron density fluctuations of $\sim 5 \%$ of the maximum concentration.

Currently, tomographic studies of the ionosphere are carried out on four chains of ground-based receivers, i.e. in Russia, Finland, Taiwan and the USA (Alaska).

Radio-tomographic data processing makes it possible to reconstruct the large-scale inhomogeneous structure of the sub-satellite region of the ionosphere. To measure the parameters of small-scale ionospheric irregularities, the time series of amplitude or phase beacon registrations should be subjected to a standard FFT (Fast Fourier Trans-form) or MEM (Maximum Entropy Method) processing procedure; as a result, power spectra are obtained, respectively, of amplitude or phase fluctuations depending on the frequency of fluctuations. For low-orbit spacecraft (with a circular orbit of $\sim 1000 \mathrm{~km}$ ), the radio beam scans the F-region $(\sim 300 \mathrm{~km})$ at a speed of $2.2 \mathrm{~km} / \mathrm{s}$. Therefore, when processing a section 
of the register program with a duration of $10 \mathrm{~s}$ and a sampling frequency of $125 \mathrm{~Hz}$, the spectral range of wavelengths from $22 \mathrm{~km}$ to $18 \mathrm{~m}$ (frequency fluctuation range from $10-1$ to $102 \mathrm{~Hz}$ ) will be covered [15].

The airborne radio beacon used for remote sensing of the ionosphere by radio scanning methods is a multichannel (usually 2 channels) transmitter that emits on the harmonics of the reference generator, thereby ensuring the coherence of signals.

Let the operating frequencies $f_{1}$ and $f_{2}$ of a coherent radio beacon

$f_{1}=m_{1} f_{0} ; f_{2}=m_{2} f_{0}$,

where $f_{0}$ is the frequency of the transmitter reference oscillator (quartz frequency stabilization is sufficient); $m_{1}$ and $m_{2}$ are integers defining the ratings of the radiated frequencies.

In this case, the phase difference of the received frequencies, reduced to the frequency $f_{1}$ $\Delta \Phi=\Phi_{1}-\left(m_{1} / m_{2}\right) \Phi_{2}$

does not depend on the "vacuum" component of the Doppler frequency shift of a moving transmitter, but is determined entirely by the influence of the ionosphere, that is, in the case of a coherent transmitter, the "vacuum" component of the Doppler frequency shift, which is several orders of magnitude higher than the "ionospheric" component, can be automatically excluded in the receiving device.

The MAYAK-150/400 instruments consist of two antennas and an electronics unit (the transmitter itself).

The antennas of the Mayak-150/400 transmitter are crossed half-wave vibrators, the directional patterns of which are oriented in the nadir.

\subsection{Measurement of ionospheric plasma parameters along the spacecraft orbit with the use of ESIP instrument}

Measurements of the main parameters of the ionospheric plasma along the spacecraft orbit are necessary both for studying the structure and dynamics of the ionosphere as a whole, and for studying individual physical processes in the ionospheric plasma and global monitoring of the ionosphere. For these purposes, the ESIP (Energy-Spectrometer of Ionospheric Plasma) equipment is used, in which several types of sensors are used to register the parameters of the ionospheric plasma. According to the principle of operation, they belong to two groups: analyzers with inhibitory potential (AIP) and Langmuir probes. Thus, the ESIP equipment includes the following measuring sensors:

- ion flux sensor (IFS);

- ion spectrum sensor (ISS);

- drift meter (DM);

- 4 potential sensors (PS);

- sensor for fast measurement of electronic temperature (ETS);

- flat Langmuir probe (FLP).

For all sensors, the directly measured quantity is the incoming particle flux. In the case of ions, the flux is given by the expression:

$F_{\mathrm{i}}=F_{\mathrm{i}}\left(N_{\mathrm{i}}, T_{\mathrm{i},} M_{\mathrm{i}}, \alpha, \beta, V_{\mathrm{SC}}, V_{\mathrm{D}}, U_{\mathrm{SC}}, U_{\mathrm{AI}}, U_{\mathrm{D}}\right)$,

where $N_{\mathrm{i}}, T_{\mathrm{i}}, M_{\mathrm{i}}$ are the concentration, temperature and mass of particles, $\alpha, \beta$ are the angular coordinates of the incident ion flux, $V_{\mathrm{SC}}, V_{\mathrm{D}}$ are the vectors of the spacecraft velocity and ionospheric plasma drift, $U_{\mathrm{SC}}$ is the spacecraft body potential, $U_{\mathrm{AI}}$ is the potential difference at the AIP electrodes, UD is the potential of the sensor body.

In this expression, the device controls (sets) only the potentials of the electrodes. The spacecraft speed is considered to be known. All other parameters are unknown. Since the thermal velocity of ions, as a rule, is significantly lower than the velocity of the spacecraft, 
the ion flux to any sensor can be represented as a parallel beam of ions incident on the sensor with the velocity $V=-V$ sc $U_{\mathrm{AI}}$.

In the case of electrons, the situation is reversed - the thermal velocity of electrons is significantly higher than the velocity of the spacecraft, so the flow of electrons to the sensor is isotropic.

Ionic parameters (concentration of $N_{\mathrm{i}}$, temperature $T_{\mathrm{i}}$, masses of the main ions $M_{\mathrm{i}}$, energy distribution, magnitude and direction of drift velocity $V_{\mathrm{D}}$ ) are measured using analyzers with the braking potential of the AIP or otherwise ion traps (Faraday cups). They are an inlet aperture, a flat multigrid design, and a collector. They are used to measure the dependence of the ion flux incident on the sensor aperture (ion current to the collector) depending on the potentials on the sensor electrodes. On this basis, by setting different potentials on the electrodes, sensor options are created for measuring various plasma parameters.

In the ESIP instrument based on AIP, three types of sensors are implemented, i.e. IFS, ISS and DM.

IFS is the Ion Flux Sensor, it is an AIP with a potential distribution on the electrodes that allows measuring the ion flux (concentration) of $N_{\mathrm{i}}$ ions. Used for fast (up to 1000 times per second) continuous $N_{\mathrm{i}}$ changes. The geophysical parameter determined with this sensor is the total ionic concentration of $N_{\mathrm{i}}$ ions in the range $10^{2}-10^{7} \mathrm{~cm}^{-3}$.

ISS is the Ionic Spectrum Sensor, it is an ATP with a potential distribution on the electrodes, providing measurement of the integral energy spectrum of ions (IES) in the energy range $\mathrm{E} \leq 15 \mathrm{eV}$. To measure the energy spectrum, a varying voltage must be applied to the analyzing grid within the limits sufficient to decelerate the heaviest incident ions. The range of measured values of $N_{\mathrm{i}}$ and $T_{\mathrm{i}}$ is $10^{2}-5 \cdot 10^{6} \mathrm{~cm}^{-3}$ and $3 \cdot 10^{2}-10^{4} \mathrm{~K}$, respectively. Measurements are carried out 1 time per second.

DM is the drift-meter, it is built on the basis of a IFS sensor with a "split" collector. This sensor allows you to measure the direction of the drift velocity $V_{\mathrm{D}}$, i.e. the vertical and horizontal components of $V_{\mathrm{D}}$ on the angles of arrival. Measurements are carried out 1 time per second. According to the readings of the angles of arrival, the spacecraft orientation relative to its velocity vector can be determined within $\pm 45^{\circ}$ in pitch and $\pm 45^{\circ}$ in yaw with an accuracy of $\pm 1^{\circ}$. This calculation can be done by the built-in microprocessor and displayed on board in real time.

For maximum efficiency of the device operation, the spacecraft must be oriented along the vector of the incident plasma flow (along the spacecraft velocity vector).

Measurement of the angles of arrival and the energy spectrum of ions makes it possible to determine the full vector of the ionospheric plasma drift velocity, the knowledge of which in combination with the calculated value (or measured in the presence of a magnetometer on board) of the magnetic field will make it possible to calculate the full vector of the constant electric field in the ionosphere.

The parameters of the electronic component of the ionospheric plasma $\left(N_{\mathrm{e}}\right.$ concentration and $T_{\mathrm{e}}$ temperature) are measured using various Langmuir probes.

The electronic temperature sensor ETS is a modified Langmuir probe and measures the electron temperature $\left(T_{\mathrm{e}}\right)$ by the method of high-frequency electron temperature probing. In addition, the potential of the spacecraft body is determined from its readings. The high frequency electron temperature probe is a flat four-electrode differential symmetric design. Each of the 4 collectors is surrounded by a guard electrode, which lowers the parasitic capacitance and increases the measurement frequency. In addition, to increase the resistance to external interference, the electrodes are cross-connected in pairs. A sinusoidal modulated voltage (no more than $500 \mathrm{mV}$ ) with a frequency of less than $50 \mathrm{kHz}$ is supplied to the working collector according to a special program. Due to the nonlinearity of the 
current-voltage characteristic "probe-plasma", the floating potential of the collector changes, the value of which is determined by the temperature $T_{\mathrm{e}}$.

If a sinusoidal voltage with a given amplitude $A$ and frequency $f$ is applied to the collector of the $T_{\mathrm{e}}$ sensor, then the potential of the collector relative to the plasma will change by an value that is a known function of the electron temperature and the amplitude of the applied sinusoidal voltage: $\mathrm{d} U=\mathrm{d} U\left(T_{\mathrm{e}}, A\right)$.

$\mathrm{d} U=\left(k T_{\mathrm{e}} / e\right) \times \ln I_{0}\left(\mathrm{e} A / k T_{\mathrm{e}}\right)$,

where $e$ is the electron charge, $k$ - the Boltzmann constant, $T_{\mathrm{e}}$ is the electron temperature, $I_{0}$ is the modified Bessel function of the first type of zero order. Since the amplitude $A$ is known, from this formula we can calculate $T_{\mathrm{e}}$ from the inverse relation $T_{\mathrm{e}}=$ $T_{\mathrm{e}}(\mathrm{d} U, A)$.

The FLP sensor (flat Langmuir probe) uses the method of the classical Langmuir probe and measures the energy distribution of electrons, from which the Ne concentration and the electron temperature Te are determined during ground processing.

The range of $k T_{\mathrm{e}}$ values measured by the ETS and FLP sensors is $0.1-5.0 \mathrm{eV}$. The measurement frequency for the ETS sensor is not less than $1 \mathrm{kHz}$, for the FLP sensor it is 3 $\mathrm{Hz}$.

The ESIP equipment sensor unit must be installed on a rod that removes it at a distance of at least $0.5 \mathrm{~m}$ from the spacecraft structural elements. In this case, it must be oriented with its axis along the spacecraft velocity vector. In this case, the sensor unit will be in the oncoming plasma flow.

\section{Monitoring of electromagnetic waves with the use of VLF - Wave Probe instrument}

As it is well-known, outer space is a medium in which electromagnetic radiation propagates and is generated in a very wide frequency range, ranging from permanent magnetic and electric fields, up to X-and gamma rays. The range from constant fields to the first tens of megahertz is specific for near-Earth space (NES); these fields are associated with the plasma shells of the Earth - the magnetosphere and the ionosphere. The restructuring of the structure of plasma shells, the development of dynamic processes in them lead to the appearance of a complex of electromagnetic phenomena recorded both in the NES and on the Earth's surface.

In turn, a number of natural electromagnetic phenomena occurring in the Earth's atmosphere contribute to the electromagnetic fields recorded in the magnetosphere (as a rule, strongly modified by the ionosphere). A classic example is the radiations recorded by satellites that arise during lightning discharges and are called whistling atmospherics.

Organization of long-term global monitoring of electromagnetic radiation in the range from constant fields to tens of kilohertz is one of the methods for controlling events (natural and anthropogenic) in near-earth space.

The main tasks of electromagnetic monitoring are:

- measurements of background electromagnetic radiation of the VLF frequency range in the NES;

- revealing the correlation of the radiation detected on satellites with extreme phenomena and processes on the ground and in the depths of the Earth (typhoons, earthquakes, powerful explosions, energy catastrophes, etc.); anthropogenic electromagnetic impact on the ionosphere and magnetosphere;

- identification of medico-biological problems of the impact of natural and anthropogenic electromagnetic fields of the considered frequency range.

The tasks solved on the basis of measurements of quasi-constant electric fields and the intensity of ULF-VLF radiation in this project include: 
- study of the dynamics of ionospheric - magnetospheric plasma under various conditions in the solar wind.

- observation of the effect of electric fields on the processes of precipitation of energetic particles into the ionosphere and other auroral and subauroral phenomena.

-

- observation of the mechanisms of formation of thin electromagnetic and plasma structures in the ionosphere and magnetosphere (electrostatic field jumps, Alfven vortices, etc.)

- observation of electromagnetic and plasma responses in the ionosphere and magnetosphere of the Earth to the formation and development of phenomena in the lower atmosphere and lithosphere associated with intense effects on them of various sources of natural origin (typhoons, earthquakes, etc.).

The use of a new type of spacecraft orbit in the "Ionosphere" project will make it possible to carry out long-term measurements in the selected power tube and, on the basis of these simultaneous measurements on two spacecraft, to separate the spatial and temporal variations of quasi-constant electric fields and the intensity of ULF-VLF radiation, which is fundamentally important for solving the problems formulated above.

Measurements of electric fields on board satellites are mainly carried out using the double probe method. This method is characterized by simplicity of implementation, reliability, versatility, and sufficiently high accuracy. To implement a measurement using this method, it is necessary to measure the potential difference between two points in space. By measuring the potential difference $\Delta U_{\mathrm{x}}$ between two bodies spaced apart in space, you can calculate the field strength between them, dividing it by the length of the measurement base $d$ :

$$
E_{\mathrm{x}}=\Delta U_{\mathrm{x}} / d \text {. }
$$

If to use several pairs of sensors in mutually perpendicular directions, you can measure 3 components of the electric field and also get the field vector. This method can be used to measure quasi-constant and alternating electric fields in the range from zero $\mathrm{Hz}$ to several tens of kilohertz.

In this case, the dynamic range of the measured values must be ensured for a quasiconstant field:

- Ex $\sim 0.1-300 \mathrm{mV} / \mathrm{m}$,

- Ey 0.1-700 mV/m,

- $\mathrm{Ez} \sim 0.1-300 \mathrm{mV} / \mathrm{m}$.

Dynamic range for variable field $\sim 1 \mu \mathrm{V} / \mathrm{m}-10 \mathrm{mV} / \mathrm{m}$.

The selected dynamic range of changes in the measured values is due to the range of scientific tasks set in the project, as well as the fact that when measuring quasi-constant electric fields in space plasma, together with the investigated fields of physical nature, the induced field $\boldsymbol{E}=\boldsymbol{V} \times \boldsymbol{B}$ is also measured, associated with the movement of probes in the external geomagnetic field., as well as the corotation field, the values of which are much higher than the investigated parameters. Subsequently, these fields are removed by digital filtering methods.

The required rate of interrogation of the measured values is at least 1 time per second, which is necessary for a spatial resolution of $\sim 10 \mathrm{~km}$ to study the mechanisms of the formation of fine structures.

The required measurement accuracy of the electric field components for the tasks solved in the project, $\Delta E_{\mathrm{x}, \mathrm{y}, \mathrm{z}}=2 \mathrm{mV} / \mathrm{m}$. It is determined by several factors that introduce errors in the measured values for $\Delta E_{\mathrm{x}, \mathrm{y}, \mathrm{z}}$, respectively. In particular, the zero drift at maximum temperature changes gives for $\Delta E_{\mathrm{x}, \mathrm{y}, \mathrm{z}}=\Delta E_{\mathrm{y}}=\Delta E_{\mathrm{z}}=0.5 \mathrm{mV} / \mathrm{m}$. With an orientation accuracy of $1^{\circ}-1.5^{\circ}$ in the roll and pitch angles and $2^{\circ}-2.5^{\circ}$ in the yaw angle, the maximum error due to the deviation of the satellite coordinate system from the orbital is 
less than $0.1 \mathrm{mV} / \mathrm{m}$ for the $\mathrm{X}$ - and $\mathrm{Y}$ - components and $0.5 \mathrm{mV} / \mathrm{m}$ for the $\mathrm{Z}$ - component at maximum roll angles. Errors due to emission currents (mainly photocurrents) arising from the inhomogeneity of the probe surface, $\Delta E<0.5 \mathrm{mV} / \mathrm{m}$. Errors due to the deviation of the probe shape from spherical do not exceed $0.03 \mathrm{mV} / \mathrm{m}$. Thus, the maximum error in measuring the electric field does not exceed $\pm 1.5 \mathrm{mV} / \mathrm{m}$ for the $E_{\mathrm{x}}$ and $E_{\mathrm{y}}$ components and $\pm 2 \mathrm{mV} / \mathrm{m}$ for the $E_{\mathrm{z}}$ - component of the field.

The VLF - wave probe complex should provide monitoring of low-frequency wave phenomena in near-earth space in the range from fractions of a $\mathrm{Hz}$ to $20 \mathrm{kHz}$. At the same time, the equipment provides for both observation of natural electromagnetic radiation and observation of anthropogenic impact on the surrounding space.

During the experiment, the following observations should be made:

- propagation of whistling atmospherics (SA), which are packets of low-frequency waves with varying frequency (it is of interest to observe partially dispersed (BH) whistling atmospherics that have passed into the ionosphere along a short path from the Earth's surface, the passage of strong BHs is associated with the penetration of electromagnetic fields from high power sources);

- seismic-ionospheric effects in quasi-constant electric and magnetic fields, as well as in the fields of the ULF/ELF range;

- occurrence and movement of typhoons, including more likely to track typhoons in Oceania.

The VLF - wave probe complex is made in the form of 3 electronic units and a system of sensors of electrical and magnetic components, including:

- SHASH 2 instrument, designed for recording and processing a large amount of information in relatively short time intervals;

- ANCh instrument designed for smooth monitoring of spectral components of the electromagnetic field in the daily storage mode of information using a relatively small amount of information;

- IESP-3R electric field measurement unit;

- electric sensors ULCH/CNCH;

- magnetic sensors LEMI-106HS.

To convert the fields into electrical signals, the pair of electric DE sensors and LEMI106HS magnetic sensor are used.

The sensors are mounted on a boom with a minimum distance of any of the sensors from the spacecraft $-2 \mathrm{~m}$ and at a distance from possible radiation sources in the frequency range of the receiving band of the sensors.

The IESP-3R instrument is an analogue electric field meter in a wide frequency band that measures components of the electric field by the double probe method in a wide frequency band of $0-1 \mathrm{MHz}$.

\section{Monitoring of space weather factors with the use of SPER/1, SG/1 and GALS/1 instruments}

As noted above, one of the factors determining the state of the ionosphere is charged particle fluxes, mainly electrons and protons of supra-thermal and high energies, falling from the tail of the magnetosphere, radiation belts, as well as energetic particles of solar and galactic cosmic rays. The fluxes of these particles in near-Earth space are mainly due to the factors of geomagnetic and solar activity, the so-called space weather. Therefore, a necessary condition for monitoring the state of the ionosphere is measuring variations of charged particle fluxes in near-Earth space in connection with Geo and Solar activity. 
$\mathrm{a}$
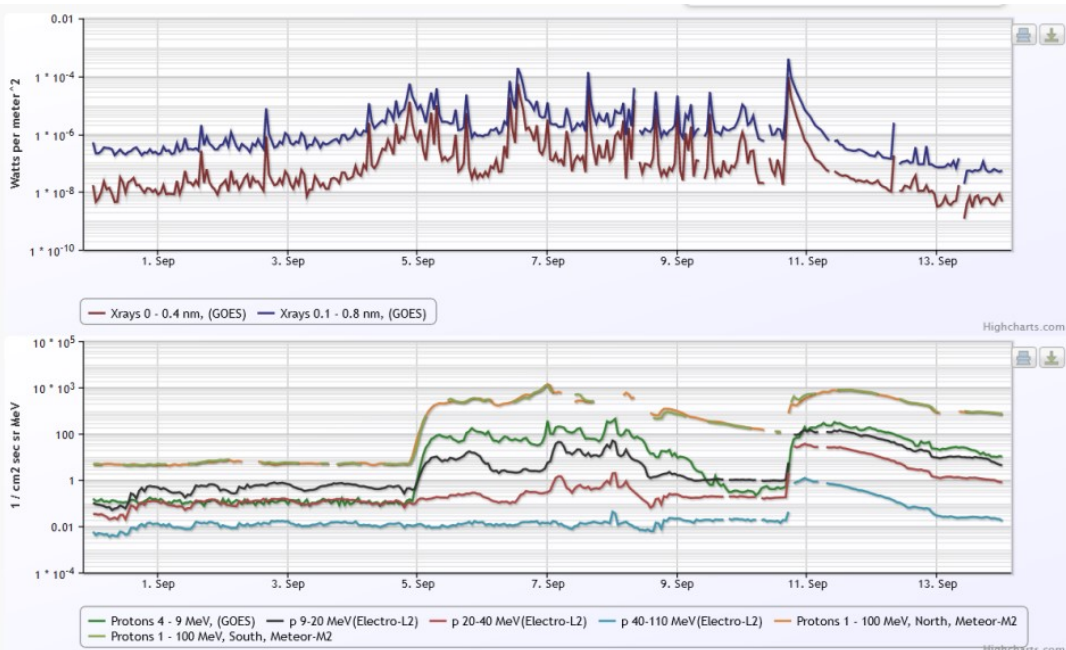

$\mathrm{b}$

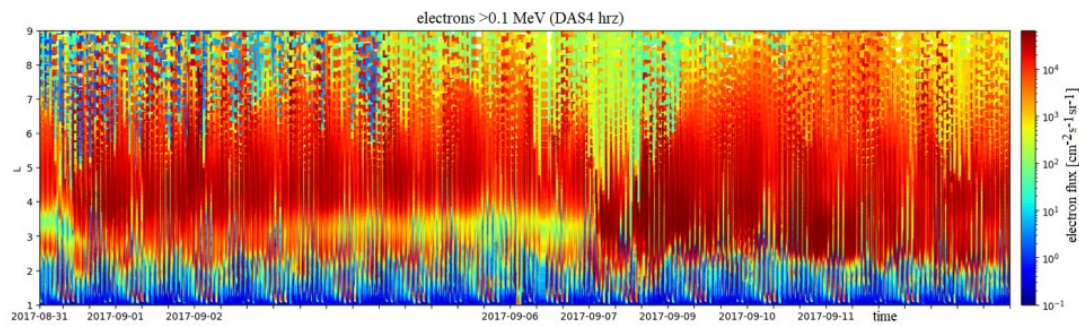

c
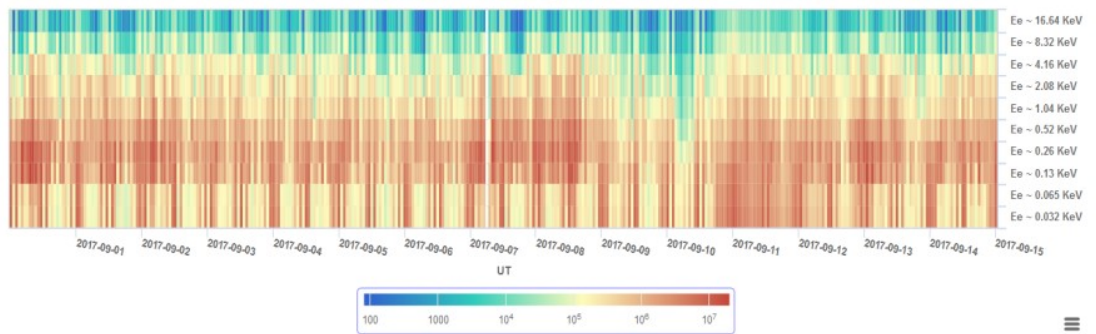

$\equiv$

d

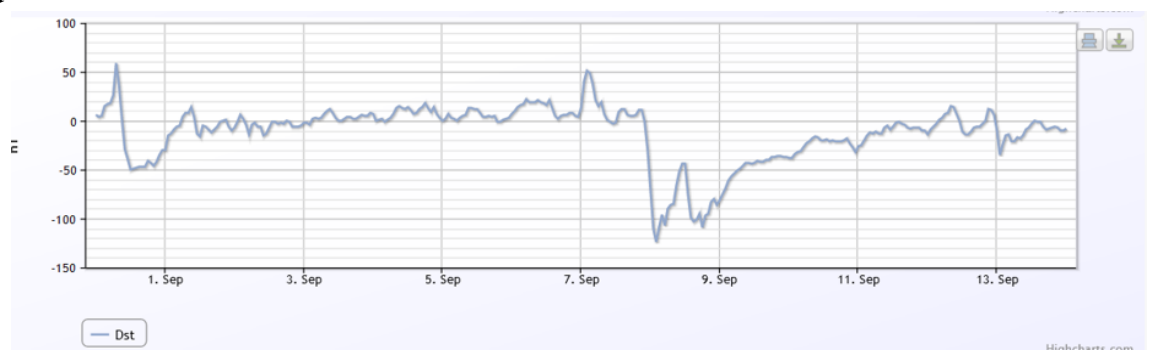

Fig. 3. Event detected $1-15$ September, 2017. Time profile of the intensity of X-rays from the sun according to GOES data (a, upper panel), SCR fluxes in the GSO and in the polar cap according to GOES data, Electro L2, SKIF instrument, Meteor, SCR instrument (a, lower panel), electron fluxes in ERBs according to Meteor data, MSGI instrument (b), spectrogram of auroral electron fluxes, Meteor-MSGI (c), time variation of $\mathrm{D}_{\mathrm{st}}(\mathrm{d})$. 
As examples of variations in fluxes of energetic charged particles of different types depending on the level of solar flare activity are shown in Fig. 3. It can be seen from the figure that after an intense solar flare in the second half of the day on September 10, 2017, increases in solar cosmic ray (SCR) fluxes are observed in the polar cap and in the geostationary orbit. At the same time, the fluxes of energetic electrons in the radiation belts and in the auroral region significantly increase after the noticeable $D_{\text {st }}$ variation on September 8, which, in turn, may have been inspired by solar flares that took place on September 5-9, which are also associated with increases in SCR fluxes on September 5-9.

\subsection{Monitoring of charge particle fluxes with the use of SPER/1 instrument}

It should be noted that the measurements of the fluxes of supra-thermal and energetic electrons on the Meteor and Electro-L2 satellites were carried out using the SKIF instrument, which is a prototype of the SPER/1 instrument, which is supposed to be used for similar measurements in the Ionosphere missions.

The SPER/1 instrument is designed to measure fluxes and differential energy spectra of low-energy electrons and protons in the energy range $0.1-20 \mathrm{keV}$, as well as charged particles of medium and high energies, i.e. electrons in the energy range $\sim 0.15-10 \mathrm{MeV}$ and protons in the energy range $\sim 2-160 \mathrm{MeV}$. The integral spectrum of $\alpha$-particles of $\mathrm{MeV}$ energies is recorded separately. The count rates should be measured at least once a second, and the total spectrum acquisition time should be no more than $20 \mathrm{~s}$.

The SPER/1 instrument is structurally a complex of detector assemblies providing detection of charged particle fluxes mentioned above, united by a common power supply and control interface. The detector assemblies include:

- unit of a medium-energy spectrometer (UMES);

- unit of a low-energy spectrometer (ULES);

- unit of a gas-discharge detector (UGD).

The detector assemblies are located in one direction on the front wall of the instrument and during the spacecraft flight must be directed to the nadir. The information from the detector units should go to the data processing unit, where it is processed and accumulated for transmission to the onboard complex for payload control and scientific data collection BKUSNI.

The detecting units are switched on independently of each other by control commands, which gives a certain flexibility in controlling the device. The sampling rates of detectors, telemetry collection parameters and some other device settings can also be changed.

The units of the low-energy spectrometer are a scanning electric separator in the form of axially symmetric spherical surfaces that focus the flows of charged particles on the input window of the secondary electron multiplier. Electron and proton detectors differ only in the polarity of the separator connection and the overclocking potential between the separator and the wind turbine. In the "default" mode, the full scan cycle for energies from $100 \mathrm{eV}$ to $20 \mathrm{keV}$ takes about $10 \mathrm{~s}$, but this setting can be changed by control commands depending on the current task and the total detector load.

The unit of the medium-energy spectrometer is made according to the classical scheme of a semiconductor telescope with three silicon detectors with thicknesses of 40,500 and $1000 \mu \mathrm{m}$, respectively, which, among other things, ensures the continuity of the data obtained with the data from the payload of meteorological spacecraft.

In the gas-discharge detector unit the SBM20-1 detector is used. Its readings are used as reference values of fluxes in the upper energy ranges. The detector is located behind the 2$\mathrm{mm}$ protection of the instrument front wall, normally to the axes of view of the remaining detecting units. 


\subsection{Monitoring of gamma quantum fluxes with the use of SG/1 instrument}

To monitor the gamma rays from the Earth's atmosphere and control the solar flare activity in the hard X- and gamma ray ranges, the SG/1 instrument is used, which measures the fluxes and differential energy spectra of hard X- and gamma rays in the near-Earth space in the energy range $0.02-10.0 \mathrm{MeV}$ at an energy resolution of $7.2 \%$ on the Cs- 137 line $(0.662$ $\mathrm{MeV})$. The effective area of the instrument for gamma ray detection is $\sim 50.0 \cdot \mathrm{cm}^{2}$. In the normal position, the instrument detector should be directed by the entrance window into the nadir. In this case, a viewing angle will be of at least $\sim 50.0 \cdot \mathrm{cm}^{2}$.

A CsI(Tl) scintillation crystal of $8 \mathrm{~cm}$ thick and $8 \mathrm{~cm}$ in diameter, viewed by a photomultiplier tube (PMT) of the Hammamatsu R1307 type, should be used as a detector in the SG/1 instrument. The instrument allows separating events associated with the detection of gamma quanta and neutrons in a CsI (Tl) crystal. The main mode of using the instrument is to carry out continuous measurements for a long time. The instrument provides measurement of count rates in 16 channels, differing in the values of energy release in the detector crystal, as well as regular transmission of three 256-channel spectra, collected separately in the low-energy region, in the hard part of the range, as well as energy release spectra corresponding to the detection of fast neutrons. In addition, the instrument has the ability to transmit a part of the primary information containing the amplitude and the exact time for each case of interaction in the detector, i.e. the so-called "event-by-event" recording. Data of this type can be generated both regularly and by the burst trigger formed at the moment the detector count rate increases.

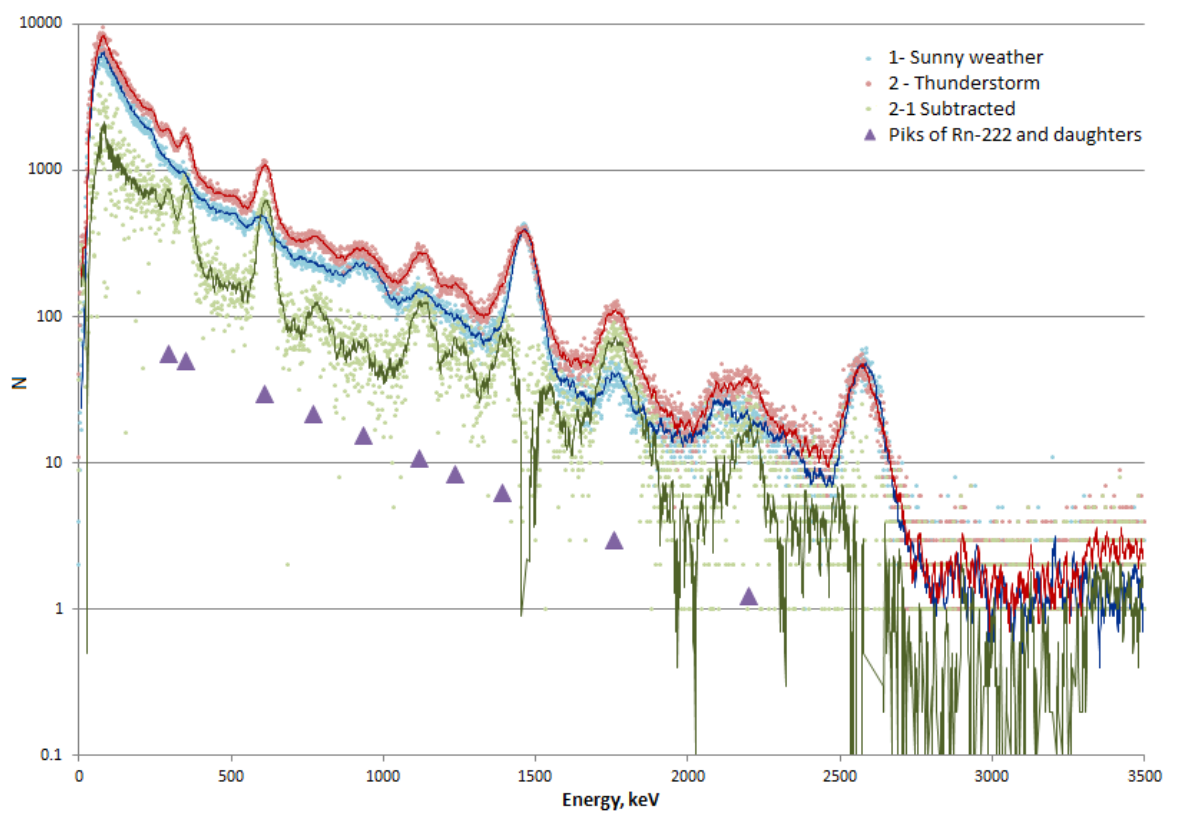

Fig. 4. Energy spectrum of atmospheric gamma rays detected on July 27, 2015 in the Moscow region during a thunderstorm and in clear weather.

Thus, scientific data from the SG/1 instrument are transmitted by information packets ("frames") of two types, i.e. "main regular information package" and "event by-event array". The size of the data field of each of them is 2032 bytes. "event by event array" frames can be generated regularly or by a burst trigger, while the internal structure of the frame does not change, but a different value of the start and end markers is used. Sampling 
rate is $1 \mathrm{~Hz}$ in background and $1 \mathrm{kHz}$ in burst mode. Sweep time, i.e. total time of energy spectrum measurement is $0.1-10 \mathrm{~s}$.

The data volume transmitted by the SG/1instrument can be adjusted using control commands that change the frequency of formation of packets of one type or another, the thresholds of the burst trigger, and other adjustable parameters. The daily quota for the SG/1 instrument is $280 \mathrm{MB}$.

As an illustration of the capabilities of the SG/1 instrument as a gamma spectrometer, we can consider the results of measurements using a laboratory prototype of the instrument, which in composition, including the detector and electronics, is fully equivalent to the flight model. In particular, the spectra of atmospheric gamma ray background at sea level, measured during a thunderstorm and for clear weather are shown in Fig. 4 [16]. The spectra show many lines of gamma rays. The background lines K-40 $\left(E_{\gamma}=1.46 \mathrm{MeV}\right)$ and Tl-208 $\left(E_{\gamma}=2.6 \mathrm{MeV}\right)$ are visible with the same intensity during a thunderstorm and in clear weather. Other lines are associated with the isotope of radon $\mathrm{Rn}-222$, which in turn is a daughter isotope of Ra-226. As is known, the lifetime of the isotope Rn-222 3.9 days makes it possible to penetrate a sufficient number of radioactive atoms into the atmosphere. After a series of decays of Rn-222, short-lived isotopes are formed, in particular Bi-214 with a half-life of $\sim 20 \mathrm{~min}$, which give the observed lines of gamma rays. Gamma ray lines Rn222 are visible at all observation intervals, but their amplitude is much greater during thunderstorms than at intervals corresponding to clear weather. The spectrum obtained by subtracting the spectrum measured during clear weather from the spectrum measured during a thunderstorm is also shown in Fig. 4. It shows that all the additional gamma rays detected during a thunderstorm are due precisely to $\mathrm{Rn}-222$ radon and its daughter isotopes.

\subsection{Monitoring of galactic and solar cosmic ray fluxes with the use of GALS instrument}

Monitoring of fluxes of high-energy particles, i.e. galactic and solar cosmic rays (GCR and SCR) is carried out with use of the GALS/1 instrument, which should measure the flux density of protons with energies of $>600 \mathrm{MeV}$ in three integral energy ranges. The instrument is a radiation detector based on a Cherenkov counter. The selection of energy ranges is provided by threshold discrimination of the detector output pulses. The operating mode of the instrument is continuous, while the data is read once per second at a frequency of $1 \mathrm{kHz}$.

The dependence of the effective detection area on the energy of the isotropic proton flux is such that at a proton energy of more than $\sim 800 \mathrm{MeV}$, it weakly depends on the energy and amounts to $\sim 36 \mathrm{~cm}^{2}$. To ensure a lower energy detection threshold with a sufficient effective area, the problem arises of separating the useful signal against the background of the dark current pulses of the multiplier itself. The requirement for the signal-to-noise ratio is reduced to the requirement that the counting rate of the detector, due to the detection of particles when measuring small (up to 5 particles $/ \mathrm{cm}^{2} \mathrm{~s}$ ) fluxes of GCR particles, is at least an order of magnitude higher than the counting rate due to dark current pulses. This requirement is met by using a low-noise photomultiplier tube and ensuring its optimal operation, taking into account the sensitivity of the electronic signal processing circuit. Based on the experience of previous similar experiments, when using a low-noise photomultiplier, it can be set at an energy level of $600 \mathrm{MeV}$. A decrease in the detection threshold can lead to an avalanche-like increase in the contribution of the photomultiplier noise pulses when operating conditions change.

The use of data from the GALS/1 spectrometer throughout the spacecraft's orbit, in particular, when it operates in the zone of the outer radiation belt, can be difficult due to the contribution of background electron radiation to its counts. To take into account the 
contribution of background radiation and, accordingly, to expand the possibility of using the measurement data of the GALS/1 spectrometer, additional measures must be taken. The level of sensitivity to a signal from $600 \mathrm{MeV}$ protons corresponds to a signal from electrons with an energy of about $1.5 \mathrm{MeV}$. The protective casing of the sensor prevents the main flux of electrons with energies up to $5-6 \mathrm{MeV}$ from entering the radiator, but it cannot completely remove the electronic background. Cutting off the electronic background can be achieved only by lowering the sensitivity of the multiplier signal registration circuit, which will lead to a simultaneous increase in the energy threshold for the detection of space radiation. To take into account the contribution of the electronic background during the semantic processing of information, an additional 2 thresholds for particle registration were introduced into the registration circuit of the photomultiplier signal of the Cherenkov detector due to the introduction of additional threshold discriminators with higher response levels. An aggregate analysis of the counts of simultaneous measurements in all channels will make it possible to exclude the contribution of the background radiation and obtain additional information on the fluxes of relativistic electrons in the NES.

\section{Monitoring of atmospheric ozone with the use of the Ozonometer instrument}

The measurement of the total content of ozone and other gases in the Earth's atmosphere has been carried out by ground-based and satellite methods for a long time. With the help of ground-based measurements, a large array of data has been obtained, which is used to solve various scientific and applied problems in the physics and chemistry of the Earth's atmosphere. However, such measurements are of a local nature and cannot give a complete picture of the ozone distribution. Since the mid-1960s, satellite methods have begun to play an increasing role in obtaining information on the ozone content, primarily a method based on the interpretation of measurements of reflected and scattered solar radiation in the ultraviolet (UV), visible and infrared (IR) spectral regions. The possibility of obtaining practically global information on the concentration of gaseous components of the atmosphere (including ozone) using satellite methods made these measurements extremely important in the study of various aspects of ozone dynamics, its relationships with various atmospheric parameters, changes in solar activity, in the study of long-term trends and factors, causing them.

Thus, to date, prerequisites have been created for the development and practical use of integrated methods of satellite monitoring of the atmosphere for solving regional and global problems of monitoring atmospheric pollution, interregional and transboundary transport of pollution, assessing the impact of these processes on climate change. Three main methods of remote sensing of the Earth's ozone layer can be distinguished: the emission method based on measurements of the Earth's own radiation and the atmosphere, the backscatter method based on measurements of ultraviolet solar radiation scattered by the atmosphere, and the absorption method associated with measuring the transparency of the atmosphere in the direction of an Earth satellite - a source of radiation (Sun or stars).

Measurement of radiation in these methods is carried out in nadir or at different angles to the nadir. According to the direction of the observation line of the equipment relative to the nadir, the measurements are conventionally divided into limb and nadir measurements.

In the case of the nadir scheme, the outgoing radiation of the atmosphere is measured in the vertical direction or at different angles to the vertical. With limb measurements, the observation line is directed towards the Earth's horizon. Due to the large length of the beam path in the atmosphere, these measurements make it possible to determine, along with ozone, the content of other small gas components in its upper layers. 
In the Ozonometer instrument, the backscattering method (nadir observation) is used for determining the ozone content in the atmosphere. Its essence is as follows. The shortwavelength part of solar radiation, as noted earlier, undergoes noticeable scattering by molecules and fluctuations in the atmospheric density. The scattering of radiation obeys Rayleigh's law, according to which the intensity of the scattered radiation is inversely proportional to the wavelength to the fourth power and is distributed in such a way that it is twice as large in the direction of propagation as in the transverse direction. The ozone content even at the maximum of its altitudinal distribution is much less than the concentration of the main components of the atmosphere. Therefore, the absorption of sunlight in the ozone bands occurs gradually, as it "deepens" into the atmosphere. Radiation penetrating the atmosphere causes a noticeable flux of backscattered UV radiation. Of course, the depth of penetration of sunlight into the atmosphere depends on the absorption capacity of ozone at a given wavelength. In the region of the main Hartley absorption band, the sun's rays are completely absorbed in the ozone layer, the intensity of outgoing UV radiation in this part of the spectrum is determined by the solar radiation scattered by the upper atmosphere and absorbed by ozone.

In the region of weaker absorption of the Higgins band, solar radiation with weakening reaches the Earth's surface. In this part of the spectrum, the outgoing radiation is determined by the scattered atmosphere and solar radiation reflected from the Earth's surface and its cloud cover.

Calculations show that in the wavelength range of $0.25-0.3 \mu \mathrm{m}$, UV radiation from the Sun is absorbed in the ozone layer without reaching the Earth's surface, and each of the wavelengths in this region has a certain scattering depth and a rather sharply pronounced relatively narrow effective scattering layer. The shorter the wavelength, the higher its "effective layer" is. This portion of the spectrum can be used to determine the vertical distribution of ozone.

UV radiation in the spectral range $0.3-0.34 \mu \mathrm{m}$ penetrates into the lower layers of the atmosphere. Scattering at these wavelengths captures practically all dense layers of the atmosphere from the Earth's surface to an altitude of $60 \mathrm{~km}$. This wavelength range is used to determine the total ozone content in the atmosphere.

The total scattered radiation from all layers determines the intensity of UV solar radiation scattered by the Earth's atmosphere. The ultimate goal of the method is the distribution of ozone in the atmosphere according to spectral measurements obtained from the satellite.

Thus, the main task of the experiment with the Ozonometer instrument is the global and continuous measurement of the ozone content in the Earth's atmosphere based on spectroscopic measurements from two strong absorption bands - the Hartley band (ozone absorption band in the range $200-300 \mathrm{~nm}$ with a maximum of about $255 \mathrm{~nm}$ ) and the Higgins band (a system of absorption bands with alternating minima and maxima in the range $300-360 \mathrm{~nm}$ ). Absorption in the Chappuis band (band $450-650 \mathrm{~nm}$ ) is weaker, but it can also be used to study the gaseous composition of the atmosphere from satellites, provided that the spectrometer sensitivity is sufficiently high. Also, by the ultraviolet and visible spectrum of the scattered radiation of the Sun, it is possible to determine the concentrations of other important gas components: $\mathrm{NO}, \mathrm{NO}_{2}, \mathrm{NO}_{3}, \mathrm{SO}_{2}, \mathrm{BrO}, \mathrm{H}_{2} \mathrm{CO}$ and others with absorption bands in the studied range. For example, nitrogen oxides $\mathrm{NO}_{\mathrm{X}}(\mathrm{NO}$, $\mathrm{NO}_{2}, \mathrm{NO}_{3}$ ) play an important role in atmospheric chemistry.

The result of measurements using the Ozonometer instrument will be:

- global maps of the distribution of the total ozone content in the atmosphere over the entire globe with a frequency of 1 day. Measurements are not carried out during the polar night;

- $\quad$ the same maps of nitrogen oxides (NO2); 
- $\quad$ maps of excess content of $\mathrm{NO} 3, \mathrm{H} 2 \mathrm{CO}, \mathrm{SO} 2$ over the natural level;

- products of the generalization of these measurements (climatology, changes from year to year, long-term trends, etc.).

To solve the set tasks, it is proposed to create a spectrometer for the UV and visible radiation range. The spectrometer assumes measurements in a wide wavelength range from 0.3 to $0.8 \mu \mathrm{m}$ with a spectral resolution of 0.4 to $0.6 \mathrm{~nm}$. The spectrometer is developed on the basis of a reflective diffraction grating using a symmetric crossed Czerny-Turner scheme. Observations are carried out in nadir. Solar radiation reflected from the Earth's surface, i.e. Twice passed through the atmosphere, it hits the rotating mirror, passes through the entrance telescope, which creates an optical beam of the required diameter, and is fed to the entrance slit of the analyzer with a size of 5 - 10 by 1000 microns. After the slit, the light hits a collimating mirror, which forms a parallel beam. This beam is directed to a diffraction grating (600 lines $/ \mathrm{mm})$, the main element of the spectrometer. After the grating, the diffracted light hits the second mirror, which focuses the first-order spectrum in the detector plane. The detector is a Toshiba silicon-based CCD array with a spectral range of $200-1100 \mathrm{~nm}, 3648$ pixels, and a pixel size of $8 \times 200 \mu \mathrm{m}$.

The design of the developed instrument provides the presence of instantaneous rectangular fields of view with angular dimensions within $1^{\circ}$, elongated in the direction perpendicular to the direction of movement of the vehicle, which at an orbital altitude of $600 \mathrm{~km}$ gives a spatial resolution on the earth's surface of $\approx 10 \mathrm{~km}$. Subsequent mapping of the distribution of ozone or other gases will represent this area as a point on the map.

\section{Conclusion}

Thus, in case of successful implementation of the Ionosphere space project, a multi-satellite constellation will be deployed for the first time, which will allow simultaneous monitoring of various regions of the ionosphere, including near-equatorial and Polar regions. In this case, both active experiments on sounding the ionosphere in order to determine the parameters of the ionospheric plasma, in particular, obtaining a three-dimensional picture of the electron concentration distribution, and passive measurements of radiation scattered in the atmosphere from ground-based and orbital transmitters will be carried out. In addition, comprehensive monitoring of space weather factors will be carried out, including control of the magnetic-wave situation, fluxes of energetic charged particles and gamma-quanta in near-Earth space. A separate range of tasks is associated with monitoring atmospheric ozone in connection with geomagnetic and solar activity.

The results to be obtained during the implementation of the "Ionosphere" mission should provide important scientific information about dynamic processes in the "atmosphere - ionosphere - magnetosphere" system, "wave-particle" interactions, acceleration of charged particles, possible nonlinear effects in the ionosphere and magnetosphere. plasma. Also, the implementation of this project will be of great practical importance in terms of monitoring the geophysical situation in near-earth space, monitoring space weather.

\section{References}

1. E.L. Afrajimovich, E.I. Astaf'eva, O.I. Berngardt, V.V. Dem'janov, T.N. Kondakova, O.S. Lesuita, B.G. Shpynev, Izv. Vuzov, Radiophysica (Russ.) 47, 509 (2004)

2. E.L. Afrajimovich, E.I. Astaf'eva, Solar-Terrestrial physics (Russ.) 3, 50 (2003)

3. L.I. Mandelshtam, N.D. Papaleksi, JETPh (Russ.) 7, 659 (1937)

4. V.V. Migulin, Uspekhi Phys. (Russ.) 128, 667 (1979) 
5. Ja.L. Al'pert, Uspekhi Phys (Russ.) 71, 369 (1960)

6. G.K. Solodovnikov, V.M. Sinelnikov., E.B. Krokhmal'nikov, Distantcionnoe zondirovanie ionosphery Zemli $\mathrm{s}$ ispolzovaniem radiomajakov i kosmicheskykh apparatov (Russ.) (Nauka, Moskva, 1988)

7. V.M. Sinelnikov., S.V. Pakhomov, A.P. Glotov, G.P. L'vova, T.L. Gyljaeva, Fazovy doplerovskii metod izmerenija vysotnogo profilja electronnoy concentracii v nizhnej ionosphere s pomoschiy geophysicheskikh raket, in Issledovanija structury i volnovykh svojstv prizemnoj plasmy (Russ.), IZMIRAN, Moskva, 40 (1980)

8. L.M. Erykhimov, O.I. Maksimenko, Issledovanie ionosphernykh neodnorodnostey pri pomoschi ISZ, in Dreify neodnorodnostey v ionosphere (Russ.), Nauka, Moskva, 41 (1973)

9. L.M. Erykhimov, E.E. Mitjakova, E.N. Mjasnikov, S.V. Poljakov, A.V. Rakhlin, V.M. Sinelnikov, Izv. vuzov. Radiophysica (Russ.) 20, 1814 (1977)

10. M.A. Kolosov et al, Doklady Akademii nauk SSSR (Russ.) 241, 555 (1978)

11. Y.-H. Kuo, S. Sokolovsky, R.A. Anthes, F. Vandenberghe, Special Issue of Terr., Atmosph. And Ocean. Sci. 11(1), 157 (2000)

12. V.E. Kynitsyn, E.D. Tereschenko, Tomografija ionosphery (Russ.) (Nauka, Moskva, 1991)

13. V.N. Oraevskii, V.E. Kynitsyn, Yu.Ja. Ruzhin, E.S. Andreeva, A.H. Depueva, E.F. Kozlov, O.G. Razinkov, I.I. Shagimuratov, Geomagnetism i aeronomia (Russ.) 35, 117 (1995)

14. V.E. Kynitsyn, E.D. Tereschenko, E.S. Andreeva, Radiotomografija ionosphery (Russ.) (FIZMATLIT, Moskva, 2007)

15. Yu.G. Ivanov, V.M. Russkin, V.M. Sinelnikov, G.K. Solodovnikov, Avtomatizirovannaja systema registratcii I obrabotky amplitudno-fazovykh fluktuatcii radiosignalov, in Volnovye processy v prizemnoy plazme (Russ.), Nauka, Moskva, 61 (1988)

16. V. Bogomolov, A. Chilingarian, G. Garipov, et al., Results of TGE Study in 0.03-10 MeV Energy Range in Ground Experiments near Moscow and Aragatz, in Proceedings of International Symposium on Thunderstorms and Elementary Particle Acceleration (TEPA-2016), Cosmic Ray Division, Yerevan Physics Institute, ISPIRE C15-10-02, 37 October 2016, Nor-Amberd, Armenia (2016) 